\title{
CORRECTION
}

\section{Correction to: Magnetic Resonance Imaging of Human Olfactory Dysfunction}

\author{
Pengfei Han ${ }^{1,2,3}$ (I) Yunpeng Zang ${ }^{3} \cdot$ Joshi Akshita $^{3} \cdot$ Thomas Hummel $^{3}$
}

Published online: 18 October 2019

○) Springer Science+Business Media, LLC, part of Springer Nature 2019

Correction to: Brain Topography

https://doi.org/10.1007/s10548-019-00729-5

The original version of this article unfortunately contained a mistake. The spelling of the Yoshi Akshita name was incorrect.

The correct name should be Joshi Akshita.
The original article has been corrected

Publisher's Note Springer Nature remains neutral with regard to jurisdictional claims in published maps and institutional affiliations.

The original article can be found online at https://doi.org/10.1007/ s10548-019-00729-5.

Pengfei Han

p.han@foxmail.com

1 Faculty of Psychology, Southwest University, Chongqing, China

2 Key Laboratory of Cognition and Personality, Ministry of Education, Southwest University, Chongqing, China

3 Department of Otorhinolaryngology, Interdisciplinary Center Smell and Taste, TU Dresden, Dresden, Germany 